\title{
What Should Be Considered for Local Excision in Early Rectal Cancer?
}

\author{
Taesung Ahn \\ Department of Surgery, Soonchunhyang University Cheonan Hospital, Soonchunhyang University College of Medicine, Cheonan, Korea
}

\section{See Articles on Page 194-201}

South Korea has the highest rates of colorectal cancer worldwide. In particular, the incidence of rectal cancer in men is among the highest in the world [1]. Despite the high incidence, Korea has the lowest mortality in terms of colorectal cancer. It is thought that the diagnosis rate is high due to the opportunities for screening covered by national health insurance and the accessibility to colonoscopy. Thus, rectal cancer is often diagnosed in its early stage. Therefore, it is necessary to consider an appropriate treatment method for early rectal cancer.

Currently, the cornerstone of curative treatment for rectal cancer is total mesorectal excision (TME) [2]. TME, introduced by Heald and Ryall [3], refers to a surgery involving complete excision of the mesorectum, resulting in significant improvement in survival. TME is recommended as a standard method for rectal cancer surgery because it reduces local recurrence by excising lymphatic drainage pathways and regional metastases. However, surgery for rectal cancer involving TME may cause complications such as anastomosis, site leakage, and nerve injury. Minimal surgery can be considered if oncological stability can be achieved.

Radical resection with TME is likely to damage the surrounding nerves. This can lead to complications including urinary and sexual dysfunction, which have a major impact on quality of life. Local excision (LE), on the other hand, reduces the probability of nerve damage compared to TME, and the cancer can be completely cured with complete tumor excision. The disadvantage of

Correspondence to: Taesung Ahn, M.D.

Department of Surgery, Soonchunhyang University Cheonan Hospital, Soonchunhyang University College of Medicine, 31 Suncheonhyang 6-gil, Dongnam-gu, Cheonan 31151, Korea

Tel: +82-41-570-3654, Fax: +82-41-571-0129

E-mail: eyetoeye@schmc.ac.kr

ORCID code: https://orcid.org/0000-0001-5617-0365

(C) 2019 The Korean Society of Coloproctology

This is an open-access article distributed under the terms of the Creative Commons Attribution NonCommercial License (http://creativecommons.org/licenses/by-nc/4.0) which permits unrestricted noncommercial use, distribution, and reproduction in any medium, provided the original work is properly cited.
TME is not nerve damage alone; TME increases the postoperative morbidity and mortality and has a significant impact on postoperative recovery, treatment compliance, and efficacy (e.g., chemotherapy and radiotherapy) [4].

It is difficult to choose between the complications that can occur in radical resections such as TME and the benefits of high-quality oncological clearance of tumors and regional lymph nodes. LE has the distinct benefit of fewer complications, but there is a greater chance of recurrence.

LE can be presented as a therapeutic plan only when oncological safety can be ensured. However, oncological safety during LE is difficult to confirm even with the use of preoperative imaging studies and postoperative pathologic findings. To address this challenge, salvage surgery may be considered when recurrence is observed during postoperative follow-up. However, Morino et al. [5] reported that TME after LE has a high incidence of complications and involves risk factors requiring abdomino-perineal resection. Hopes et al. [6] found unfavorable pathology in damaged specimens after LE completion surgery, and salvage surgery decreased the disease-free survival compared to radical surgery. Therefore, it is necessary to have clear and reliable criteria when performing LE.

If oncological safety can be ensured, LE is considered a superior therapeutic option with low morbidity and complications. It is generally accepted that LE (e.g., transanal excision) results in lower morbidity and fewer complications than radical resection. Achieving oncological safety (or integrity) in LE is an important issue.

In general, indications for LE include: (1) physical examination findings such as tumor size less than $3 \mathrm{~cm}$, internal diameter less than $30 \%$, and less than $15 \mathrm{~cm}$ of dentate line; (2) radiological findings (magnetic resonance imaging or transrectal ultrasonography) within the submucosal layer and without node involvement; (3) histological findings such as well or moderately differentiated perineural invasion without lymphovascular invasion (LVI) [6].

pT2 cancer is also in the range that can be sufficiently excised via LE. However, previous studies suggest that LE cannot be recommended as a standard treatment for pT2 rectal cancers. LE for pT2 has a high recurrence rate and higher mortality rate compared to TME $[7,8]$. Lymph node metastasis is less likely in pT2 
cancer, but higher than in T1. This is thought to be the cause of regional lymphatic spreading and high mortality even with LE of pT2 rectal cancer.

The LE of T1 rectal cancer is considered a standard treatment because of its acceptable oncological safety [9-12]. The reasons for performing LE on T1 rectal cancer are because of the very low vascular or lymphatic metastasis; if they exist, their presence is locally confined. However, although T1 is less than T2, regional lymph node metastases are also found [13]. For this reason, oncological safety cannot always be ensured for LE on all T1 rectal cancers. Therefore, high-risk pT1 rectal cancer (tumor size $>3$ $\mathrm{cm}$, poor differentiation, tumor budding, LVI, submucosal invasion $\geq 1 \mathrm{~mm}$ ) shows frequent local recurrence, and this serves as a rationale for the argument that standard resection including TME should be performed even in the case of T1 $[9,14,15]$.

Regarding T1 or T2 rectal cancer after LE, the presence of remnant tumors can also be reduced through radiation. Although radiation therapy after LE reduced local recurrence for 5 years, Paty et al. reported that radiation therapy had no effect on overall recurrence but only delayed local recurrence $[16,17]$. The most important factor is the complete excision of the tumor, which leads to an increase in overall survival and decrease in local recurrence. So complete excision of the tumor rather than the effect of postoperative radiation therapy is key to prevent recurrence.

Rectal cancer limited to the submucosa can be sufficiently removed by LE when the resection margin is secured. However, even in early rectal cancer, with an indication of LE, local recurrence can take place in the case of LE.

According to Hwang et al. [18], compared with only T1 rectal cancer among early rectal cancers, the TAE group showed a significant increase in local recurrence compared to the TME group. Especially, it was shown that the risk factor for local recurrence was histologic findings, not physical examination results. This indicates that local recurrence after LE of early rectal cancer is due to the characteristics of the tumor rather than surgical technique differences.

LE procedures such as transanal excision, transanal endoscopic microsurgery, and transanal minimally invasive surgery have been developed. LE also has a sufficient resection margin. However, LE still has higher local recurrence compared to TME. Various studies have been conducted on the cause and suggest that histologic findings are important factors that have significant implications. In addition, further studies, such as those that evaluate genetic characteristics, may be helpful in selecting effective LE patient groups. However, the choice between LE and TME in early rectal cancer still needs to be carefully determined with consideration of the benefits and risks.

\section{CONFLICT OF INTEREST}

No potential conflict of interest relevant to this article was reported.

\section{REFERENCES}

1. Bray F, Ferlay J, Soerjomataram I, Siegel RL, Torre LA, Jemal A. Global cancer statistics 2018: GLOBOCAN estimates of incidence and mortality worldwide for 36 cancers in 185 countries. CA Cancer J Clin 2018;68:394-424.

2. Kapiteijn E, Marijnen CA, Nagtegaal ID, Putter H, Steup WH, Wiggers T, et al. Preoperative radiotherapy combined with total mesorectal excision for resectable rectal cancer. N Engl J Med 2001; 345:638-46.

3. Heald RJ, Ryall RD. Recurrence and survival after total mesorectal excision for rectal cancer. Lancet 1986;1:1479-82.

4. Khoo CK, Vickery CJ, Forsyth N, Vinall NS, Eyre-Brook IA. A prospective randomized controlled trial of multimodal perioperative management protocol in patients undergoing elective colorectal resection for cancer. Ann Surg 2007;245:867-72.

5. Morino M, Allaix ME, Arolfo S, Arezzo A. Previous transanal endoscopic microsurgery for rectal cancer represents a risk factor for an increased abdominoperineal resection rate. Surg Endosc 2013; 27:3315-21.

6. Hompes R, McDonald R, Buskens C, Lindsey I, Armitage N, Hill J, et al. Completion surgery following transanal endoscopic microsurgery: assessment of quality and short- and long-term outcome. Colorectal Dis 2013;15:e576-81.

7. Leijtens JWA, Koedam TWA, Borstlap WAA, Maas M, Doornebosch PG, Karsten TM, et al. Transanal endoscopic microsurgery with or without completion total mesorectal excision for T2 and T3 rectal carcinoma. Dig Surg 2019;36:76-82.

8. Xu ZS, Cheng H, Xiao Y, Cao JQ, Cheng F, Xu WJ, et al. Comparison of transanal endoscopic microsurgery with or without neoadjuvant therapy and standard total mesorectal excision in the treatment of clinical T2 low rectal cancer: a meta-analysis. Oncotarget 2017;8:115681-90.

9. Doornebosch PG, Zeestraten E, de Graaf EJ, Hermsen P, Dawson I, Tollenaar RA, et al. Transanal endoscopic microsurgery for T1 rectal cancer: size matters! Surg Endosc 2012;26:551-7.

10. Christoforidis D, Cho HM, Dixon MR, Mellgren AF, Madoff RD, Finne CO. Transanal endoscopic microsurgery versus conventional transanal excision for patients with early rectal cancer. Ann Surg 2009;249:776-82.

11. Middleton PF, Sutherland LM, Maddern GJ. Transanal endoscopic microsurgery: a systematic review. Dis Colon Rectum 2005;48: 270-84.

12. Moore JS, Cataldo PA, Osler T, Hyman NH. Transanal endoscopic microsurgery is more effective than traditional transanal excision for resection of rectal masses. Dis Colon Rectum 2008;51: 1026-30.

13. You YN, Baxter NN, Stewart A, Nelson H. Is the increasing rate of local excision for stage I rectal cancer in the United States justified?: a nationwide cohort study from the National Cancer Database. Ann Surg 2007;245:726-33.

14. Bosch SL, Nagtegaal ID. What is "good quality" in rectal cancer 
surgery? The pathologist's perspective. Recent Results Cancer Res 2014;203:41-6.

15. Bosch SL, Teerenstra S, de Wilt JH, Cunningham C, Nagtegaal ID. Predicting lymph node metastasis in pT1 colorectal cancer: a systematic review of risk factors providing rationale for therapy decisions. Endoscopy 2013;45:827-34.

16. Chakravarti A, Compton CC, Shellito PC, Wood WC, Landry J, Machuta SR, et al. Long-term follow-up of patients with rectal cancer managed by local excision with and without adjuvant irra- diation. Ann Surg 1999;230:49-54.

17. Paty PB, Nash GM, Baron P, Zakowski M, Minsky BD, Blumberg $\mathrm{D}$, et al. Long-term results of local excision for rectal cancer. Ann Surg 2002;236:522-29.

18. Hwang Y, Yoon YS, Bong JW, Choi HY, Song IH, Lee JL, et al. Long-term transanal excision outcomes in patients with $\mathrm{T} 1 \mathrm{rectal}$ cancer: comparative analysis of radical resection. Ann Coloproctol 2019;35:194-201. 